\section{Transdisciplinary Approach to Managing Hepatitis C Virus Infection in Patients at a Tertiary Care Cancer Center}

\author{
Harrys A. Torres, MD; Lillian R. Roach, RN-BC; Parag Mahale, MBBS, MPH; \\ Minas P. Economides, MD; Boris Blechacz, MD, PhD; Ethan Miller, MD; \\ Roy Borchardt, PA-C, PhD; Anis Rashid, MD; Thein H. Oo, MD; \\ Bhavarth Shukla, MD, MPH; Malik Farida, MD; Charles D. Ericsson, MD; \\ Bruno P. Granwehr, MD, MS; and Issam I. Raad, MD
}

(This commentary was presented in part at ID Week 2015, October 7-11, 2015, San Diego, California.)

Hepatitis $\mathrm{C}$ virus (HCV) infection is a major problem worldwide, with 230 million reported cases, including 2.7 to 3.9 million cases $(1.0 \%-1.5 \%)$ in the United States. ${ }^{1}$ The prevalence rates for $\mathrm{HCV}$ infection in patients with cancer range from $1.5 \%$ to $32 \% .^{2}$ Although $\mathrm{HCV}$ infection in this patient population is common, little is known about its management, and many oncologists remain unaware of current treatment options. Education of medical staff and the coordination of different specialties are essential to provide the appropriate patient stratification and best therapeutic approach to treating HCV infection in patients with cancer in the context of multiple comorbidities. Therefore, this special population of patients needs guided treatment and constant supervision. This commentary highlights the importance of treating $\mathrm{HCV}$ infection in patients with cancer and describes the educational experience with a transdisciplinary approach to treatment gained at our HCV infection clinic, the first clinic in the United States devoted to managing this infection in patients with cancer. $^{3}$

\section{Treating HCV Infection in Patients With Cancer}

Patients with cancer and HCV infection benefit from antiviral therapy for several reasons. First, persistent elevation of transaminase levels as a result of chronic HCV infection can complicate cancer treatment. ${ }^{4,5}$ Second, acute exacerbation and reactivation of chronic $\mathrm{HCV}$ infection can occur after systemic chemotherapy, which may lead to discontinuation or modification of potentially life-saving cancer treatment. ${ }^{6}$ Third, HCV treatment may halt the progression of liver disease in patients with cancer and may improve overall survival rates, as seen in patients with $\mathrm{HCV}$-associated hepatocellular carcinoma and non-Hodgkin's lymphoma, and in hematopoietic cell transplant recipients. ${ }^{2,7-10}$ Fourth, treatment of HCV infection in patients with certain cancers, such as indolent B-cell non-Hodgkin's lymphoma, has induced tumor regression and improved hematologic outcomes. ${ }^{8,9}$ Lastly, elimination of HCV infection allow make patients with cancer to be eligible for several investigational chemotherapies for which they are currently excluded.

Treating HCV infections in patients with cancer is intricate. These patients often have immunosuppression due to systemic chemotherapies and underlying hematologic abnormalities, such as neutropenia, anemia, and thrombocytopenia. Before 2013, antiviral therapy for HCV infections consisted of pegylated interferon and ribavirin, which were administered in lengthy regimens (up to 48 weeks). Many hematologic side effects were seen, including bone marrow suppression, potential exacerbation of graft-versus-host disease, and even graft failure, making such treatment inappropriate in many instances. ${ }^{10,11}$ Moreover, the rate of sustained virologic response (regarded as cure of HCV infection) with the use of these antiviral agents was low. ${ }^{2}$ The complexity
Harrys A. Torres, MD

Harrys A. Torres, MD, is an Associate Professor of Medicine in the Department of Infectious Diseases, Infection Control and Employee Health at The University of Texas MD Anderson Cancer Center in Houston, Texas, where he is also founder and director of the Hepatitis C Virus (HCV) Clinic, the first established clinic in the United States devoted to managing $\mathrm{HCV}$ infection in cancer patients.

Lillian R. Roach, RN-BC

Lillian R. Roach, RN-BC, is from the Department of Infectious Diseases, Infection Control and Employee Health, The University of Texas MD Anderson Cancer Center, Houston, Texas.

Parag Mahale, MBBS, MPH

Parag Mahale, MBBS, MPH, is from the Department of Infectious Diseases, Infection Control and Employee Health, The University of Texas MD Anderson Cancer Center, Houston, Texas.

Minas P. Economides, MD

Minas P. Economides, MD, is from the Department of Infectious Diseases, Infection Control and Employee Health, The University of Texas MD Anderson Cancer Center, Houston, Texas. 
Boris Blechacz, MD, PhD

Boris Blechacz, MD, PhD, is from the Department of Gastroenterology, Hepatology and Nutrition, The University of Texas MD Anderson Cancer Center, Houston, Texas.
Ethan Miller, MD

Ethan Miller, MD, is from the Department of Gastroenterology, Hepatology and Nutrition, The University of Texas MD Anderson Cancer Center, Houston, Texas.
Roy Borchardt, PA-C, PhD

Roy Borchardt, $\mathrm{PA}-\mathrm{C}, \mathrm{PhD}$, is from the Department of Infectious Diseases, Infection Control and Employee Health, The University of Texas MD Anderson Cancer Center, Houston, Texas.

\section{Anis Rashid, MD}

Anis Rashid, MD, is from the Department of Psychiatry, The University of Texas MD Anderson Cancer Center, Houston, Texas.
Thein $\mathrm{H}$. Oo, MD

Thein H. Oo, MD, is from the Section of Benign Hematology, The University of Texas MD Anderson Cancer Center, Houston, Texas. of managing HCV infections in patients with cancer was beyond the scope of a single specialty, and required an organized transdisciplinary approach to management. Therefore, healthcare workers involved in the care of patients with HCV infection and cancer had to undergo education and training to meet the challenges presented.

\section{A New HCV Infection Clinic}

In August 2009, infectious disease specialists, in collaboration with hepatologists, established a clinic at The University of Texas MD Anderson Cancer Center devoted to managing $\mathrm{HCV}$ infections in patients with cancer. ${ }^{3}$ News of the availability of this clinic was disseminated to the institution via departmental, divisional, and institutional resources. Oncologists at MD Anderson were informed about the clinic, and the need to refer patients to it for specialized HCV infection care was stressed. Initially, the primary oncology team screened patients for HCV antibodies, and patients with confirmed infections were seen in the clinic.

Eligible patients received standard-of-care antiviral treatment that followed the guidelines for patients without cancer. ${ }^{1}$ Patients for whom antiviral therapy was not recommended (eg, those ineligible for or unwilling to undergo treatment, or those with limited life expectancies) were referred to hepatologists for management of chronic liver disease and surveillance for hepatocellular carcinoma.

As expected with difficult-to-treat HCV infections, the involvement of many services was imperative. We recruited full-time physician assistants and nurses, and designated a primary HCV nurse. The crux of our approach to management was to involve physicians specialized in various disciplines in order to care for patients as a team. Specifically, the primary oncology team planned cancer treatment, hepatologists monitored patients for liver disease progression and managed associated complications, hematologists and psychiatrists addressed the relevant side effects of treatment, and infectious disease specialists provided clinical care and collaborated with oncologists and hepatologists to optimize antiviral therapy.

Educational activities for the clinical teams included seminars, grand rounds, intraining programs, and department presentations. We implemented these activities to train nurses, advanced practice providers, public health students, research fellows, infectious disease clinical fellows, and attending physicians in the diagnosis and management of $\mathrm{HCV}$ infection.

Since 2009, 30 nurses at MD Anderson have undergone training for the treatment of HCV infections, 12 advanced practice providers have received training on the delivery of follow-up clinical care for HCV infection and assessment of treatment side effects and compliance, and more than 600 patients and their caregivers have received education about the infection. During each clinic visit, patients are educated about and given pamphlets on HCV infection. In 2014, 4 infectious disease fellows joined the clinic as part of elective rotations. The fellows underwent core curriculum training, with responsibilities that include clinical care and participation in clinical research protocols. Furthermore, 7 research fellows who have served in the clinic have presented clinical data on $\mathrm{HCV}$ infection in patients with cancer in 39 abstracts at professional meetings and 23 articles published in peer-reviewed journals. Their findings address various aspects of HCV infection, including epidemiology, oncogenesis, complications, and treatment outcomes. Figure $1 \mathrm{~A}$ shows the roles played by team members in the $\mathrm{HCV}$ infection clinic.

From August 2009 to July 2016, $607 \mathrm{HCV}$-infected patients with cancer underwent evaluation in the MD Anderson HCV infection clinic. The HCV infection treatment algorithm used at our clinic as of July 2016 takes into account the patient's HCV genotype, cancer type and status, need for cancer treatment, and life expectancy to 
design individualized treatment plans. ${ }^{12}$ This algorithm is frequently updated to reflect changes in HCV management, which are occurring with the introduction of new direct-acting antiviral agents. Figure $1 \mathrm{~B}$ shows the current approach to caring for patients with cancer with HCV infection by the transdisciplinary teams managing our clinic.

\section{Future Plans}

Future plans for the clinic include evaluation of patient and caregiver knowledge and attitudes regarding $\mathrm{HCV}$ transmission and antiviral therapy to determine how to better assist caregivers in understanding the complexity of HCV infection in patients with cancer. Patients and caregivers must understand the importance of complying with treatment of the infection.

Other plans include development of new research protocols and standardized protocols for starting and monitoring antiviral therapy in patients with cancer. We will continue to provide significant contributions to management guidelines for

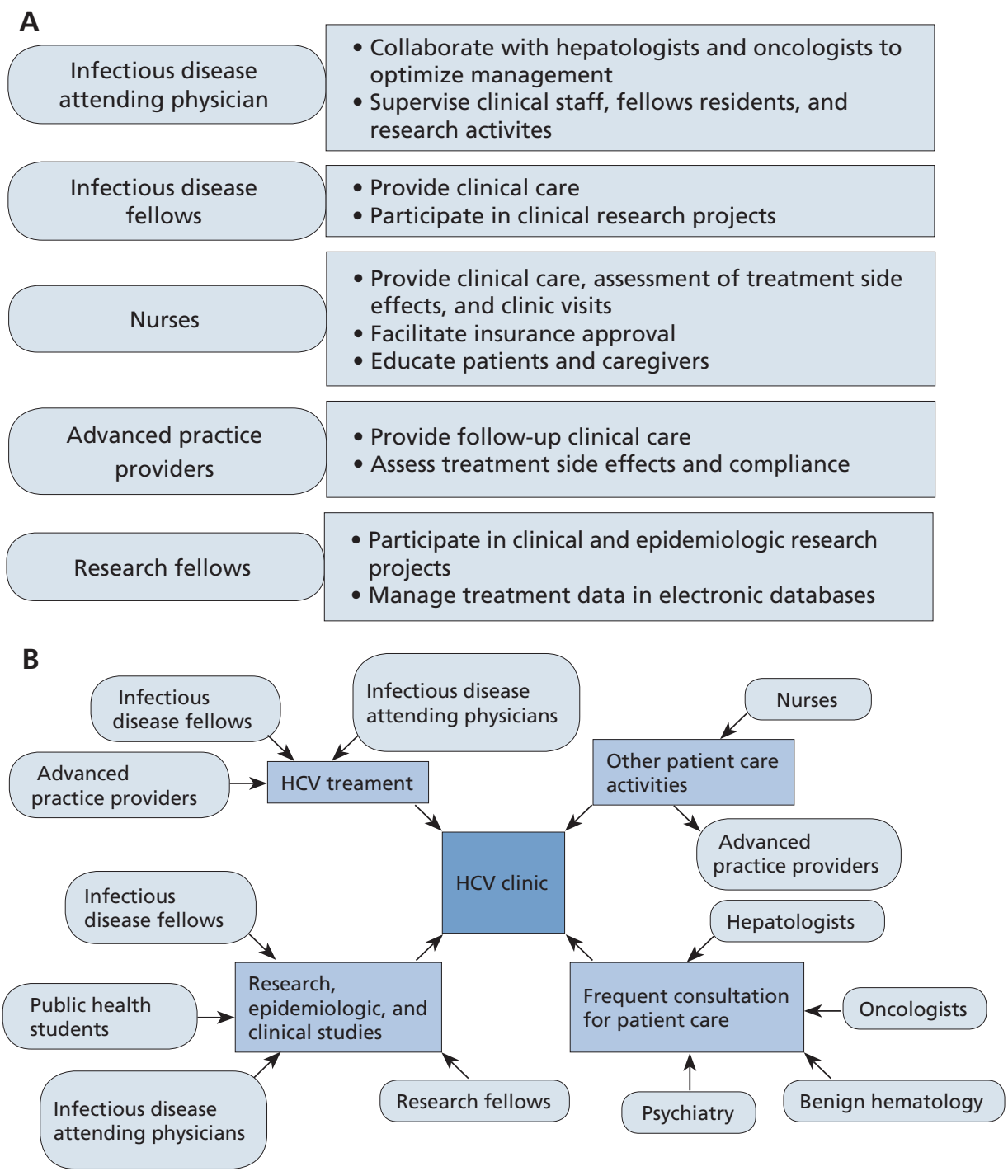

Figure 1. (A) Team members in the MD Anderson hepatitis $\mathrm{C}$ virus (HCV) clinic and their primary roles. (B) The transdisciplinary teams that manage the HCV clinic.
Bhavarth Shukla, MD, MPH

Bhavarth Shukla, MD, MPH, is from the Department of Infectious Diseases, Infection Control and Employee Health, The University of Texas MD Anderson Cancer Center, Houston, Texas.

\section{Malik Farida, MD}

Malik Farida, MD, is from the Department of Infectious Diseases, Infection Control and Employee Health, The University of Texas MD Anderson Cancer Center, Houston, Texas.

Charles D. Ericsson, MD

Charles D. Ericsson, MD, is from the Department of Internal Medicine, Division of Infectious Diseases, The University of Texas Health Science Center at Houston, Houston, Texas.

Bruno P. Granwehr, MD, MS Bruno P. Granwehr, MD, MS, is from the Department of Infectious Diseases, Infection Control and Employee Health, The University of Texas MD Anderson Cancer Center, Houston, Texas.

Issam I. Raad, MD

Issam I. Raad, MD, is Chairman, Department of Infectious Diseases, Infection Control and Employee Health, The University of Texas MD Anderson Cancer Center, Houston, Texas. 
patients with HCV infection with any type of malignancy, as recently published for hematopoietic stem cell transplant donors and recipients. ${ }^{13}$ We believe our experience can also be expanded to other areas, including centers with fewer resources and in remote locations. These can be modeled from successful programs such as the Extension for Community Healthcare Outcomes ${ }^{14}$ and similar health care outreach initiatives.

\section{Conclusions}

Our experience shows that management and treatment of $\mathrm{HCV}$ infection in patients with cancer can be optimized by implementation of a transdisciplinary approach at a tertiary care cancer center. Similar to patients with HIV infection and HCV co-infection, and those who have received a solid organ transplant, patients with cancer and HCV infection represent a specialized group of individuals with immunosuppression who need a careful, planned approach to antiviral treatment. Further, the involvement of various medical specialties can help meet their unique health care requirements. Educational efforts for these patients also provide learning opportunities for nurses, advanced practice providers, public health students, and physicians in training. We believe that the training experience offered by our institution can be replicated at other centers.

\section{Acknowledgments}

The authors thank Don Norwood for editorial assistance.

\section{References}

1. AASLD/IDSA/IAS-USA. Recommendations for testing, managing and treating hepatitis C. Available at http:// www.hcvguidelines.org. Accessed February 262016.

2. Torres HA, Mahale P, Blechacz B, et al. Effect of hepatitis $C$ virus infection in patients with cancer: addressing a neglected population J Natl Compr Canc Netw 2015;13:41-50.

3. Torres HA, Adachi JA, Roach LR, et al. Hepatitis C clinic operated by infectious disease specialists at a comprehensive cancer center: help is on the way. Clin Infect Dis 2012;54:740-742.

4. Locasciulli A, Alberti A. Hepatitis $C$ virus serum markers and liver disease in children with leukemia. Leuk lymphoma 1995;17:245-249.

5. Torres HA, Kaseb A, Mahale $\mathrm{P}$, et al. Telaprevir-containing regimen for treatment of hepatitis $\mathrm{C}$ virus infection in patients with hepatocellular carcinoma awaiting liver transplantation: a case series. J Hepatocellular Carcinoma 2014;1:109-114.

6. Mahale P, Kontoyiannis DP, Chemaly RF, et al. Acute exacerbation and reactivation of chronic hepatitis $\mathrm{C}$ virus infection in cancer patients. J Hepatol 2012;57:1177-1185.

7. Borchardt RA, Torres HA. Challenges in managing hepatitis $\mathrm{C}$ virus infection in cancer patients. World $\mathrm{J}$ Gastroenterol 2014;20:2771-2776.

8. Peveling-Oberhag J, Arcaini L, Hansmann ML, Zeuzem S. Hepatitis C-associated B-cell non-Hodgkin lymphomas: epidemiology, molecular signature and clinical management. J Hepatol 2013;59:169-177.

9. Arcaini L, Vallisa D, Rattotti S, et al. Antiviral treatment in patients with indolent B-cell lymphomas associated with HCV infection: a study of the Fondazione Italiana Linfomi. Ann Oncol 2014;25:1404-1410.

10. Kyvernitakis A, Mahale P, Popat UR, et al. Hepatitis $C$ virus infection in patients undergoing hematopoietic cell transplantation in the era of direct-acting antiviral agents. Biol Blood Marrow Transplant 2016;22:717-722.

11. Klingemann HG, Grigg AP, Wilkie-Boyd $K$, et al. Treatment with recombinant interferon (alpha-2b) early after bone marrow transplantation in patients at high risk for relapse. Blood 1991;78:3306-3311.

12. Torres HA, McDonald GB. How we treat hepatitis $C$ virus infection in patients with hematologic malignancies [published online ahead of print]. Blood, pii: blood-2016-05-718643.

13. Torres HA, Chong PP, De Lima M, et al. Hepatitis $C$ virus infection among hematopoietic cell transplant donor and recipients: American Society for Blood and Marrow Transplantation Task Force Recommendations. Biol Blood Marrow Transplant 2015;21:1870-1882.

14. Arora S, Thornton $\mathrm{K}$, Murata G, et al. Outcomes of treatment for hepatitis $\mathrm{C}$ virus infection by primary care providers. N Engl J Med 2011;364:2199-2207. 